\title{
Molecular genetic basis and prevalence of glycogen storage disease type IIIA in the Faroe Islands
}

\author{
René Santer*,1, Martina Kinner ${ }^{1}$, Ulrike Steuerwald ${ }^{2}$, Susanne Kjærgaard ${ }^{3}$, \\ Flemming Skovby ${ }^{3}$, Henrik Simonsen ${ }^{4}$, Wen-Ling Shaiu ${ }^{5}$, Yuan-Tsong Chen ${ }^{5}$, \\ Reinhard Schneppenheim ${ }^{6}$ and Jürgen Schaub ${ }^{1}$
}

${ }^{1}$ Department of Pediatrics, University Children's Hospital, Kiel, Germany; ${ }^{2}$ National Hospital, Tórshavn, Faroe Islands; ${ }^{3}$ Department of Clinical Genetics, Rigshospitalet, Copenhagen, Denmark; ${ }^{4}$ Department of Clinical Biochemistry, Statens Serum Institute, Copenhagen, Denmark; ${ }^{5}$ Department of Pediatrics, Duke University Medical Center, Durham,NC, USA; ${ }^{6}$ Department of Pediatric Hematology and Oncology, University Children's Hospital, Hamburg, Germany

Glycogen storage disease type IIIA (GSD IIIA) is caused by mutations of the amyloglucosidase gene (AGL). For most populations, none of the $A G L$ mutations described to date is particularly frequent. In this paper, we report that six children with GSD IIIA from the Faroe Islands were found to be homozygous for the novel nonsense mutation c. $1222 \mathrm{C}>\mathrm{T}(\mathrm{R} 408 \mathrm{X})$ of the $A G L$ gene. This mutation is easily detected by restriction enzyme digest with Nsil after mismatch PCR. Investigating five intragenic polymorphisms, we could show that this mutation was always associated with the same haplotype. The c.1222C $>$ T mutation could be detected on two chromosomes of another 50 unselected GSD IIIA patients of other European or North American origin which means that this mutation plays a minor role worldwide. From the fact that we are currently aware of a total of 14 GSD IIIA cases in the Faroese population of 45000 , the observed prevalence is $1: 3100$. While the novel $A G L$ mutation $c .1222 \mathrm{C}>\mathrm{T}$ was not detectable among 198 German newborns, nine out of $\mathbf{2 7 2}$ children from the Faroese neonatal screening program were found to be heterozygous for this mutation. Thus, the calculated prevalence is $1: 3600(95 \% \mathrm{Cl} 1: 700-1: 6400)$. We conclude that due to a founder effect, the Faroe Islands have the highest prevalence of GSD IIIA world-wide. The detection of the molecular defect has facilitated the diagnosis and has offered the opportunity for prenatal diagnosis in this patient group. European Journal of Human Genetics (2001) 9, 388-391.

Keywords: amyloglucosidase; glycogen debranching enzyme; glycogen storage disease; Faroe Islands

\section{Introduction}

Glycogen storage disease type IIIA (GSD IIIA, OMIM 232400) is caused by mutations in the amyloglucosidase gene $(A G L)$. Molecular genetic diagnosis is laborious because the $A G L$ gene with its coding sequence of $7.0 \mathrm{~kb}$

*Correspondence: PD Dr R Santer, Univ Children's Hospital,

Schwanenweg 20, D-24105 Kiel, Germany.

Tel.: +49 431597 1622; Fax: +49 431597 1831;

E-mail: santer@pediatrics.uni-kiel.de

Received 22 November 2000; revised 2 January 2001; accepted 11 January 2001 is relatively large and none of the approximately $30 A G L$ mutations detected to date is particularly frequent. ${ }^{1}$ Besides an unusually high frequency among North African Jews in Israel, ${ }^{2}$ a cluster of GSD IIIA cases has long been known to exist in the Faroe Islands. ${ }^{3}$ The population of this small archipelago in the North Atlantic $\left(7^{\circ} \mathrm{W}, 62^{\circ} \mathrm{N}\right)$ has its roots in the colonisation by Norwegians in the eighth century and throughout the Viking Age. Here we report the molecular basis of the Faroese GSD IIIA cases together with the results of our calculation of prevalence numbers demonstrating that the Faroe Islands have the highest prevalence of GSD IIIA world-wide. 


\section{Materials and methods \\ Subjects}

Molecular genetic studies were performed in six children (born between June 1996 and January 2000) with GSD IIIA and their relatives from five families from the Faroe Islands. The clinical signs were typical in the sense that all patients had isolated hepatomegaly. Hypoglycaemia, muscle weakness and cardiomyopathy were variable clinical signs. GSD IIIA was biochemically proven in the index case by determination of glycogen content and amyloglucosidase activity in a liver sample. DNA samples of another 50 individuals with a biochemical diagnosis of GSD IIIA of North American or European origin were investigated for comparison. Molecular genetic studies were further applied to 272 DNA samples extracted from randomly chosen anonymous Guthrie testcards from the Faroe Islands and from 198 samples from the northern German screening program for inborn metabolic diseases. These studies were approved by the ethics committees of Copenhagen, Denmark ((KF)01-201/99), the Faroe Islands and the University of Hamburg, Germany, respectively.

\section{Molecular studies}

Genomic DNA was extracted from leukocytes according to standard protocols; for the preparation from dried blood samples the E.Z.N.A. ${ }^{\mathbb{R}}$-Tissue DNA kit (Peqlab Biotechnologie, Erlangen, Germany) was used. Sense (sn) and antisense (asn) primer sequences for PCR amplification of exons 3 to 35 and the flanking intronic sequences of the $A G L$ gene were chosen from the published gene sequence (GenBank accession numbers AB035420-AB035443). ${ }^{4}$ Primer sequences used for the detection of mutations and polymorphisms reported in this paper are given in Table 1. Mutation analysis was performed according to the Thermo-Sequenase ${ }^{\mathbb{R}}$ cycle sequencing kit (Amersham/Buchler, Wenden, Germany) using ${ }^{33}$ P-radiolabelled terminators. Mutations and polymorphisms were confirmed by restrictions fragment length (RFL) analysis, eventually after PCR with a mismatch primer and digestion with the respective enzyme (Table 1 ).

\section{Results}

\section{Mutation analysis}

The novel nonsense mutation c.1222 C > T (R408X) of the $A G L$ gene was found in the homozygous state in all six GSD IIIA patients from the Faroe Islands (Figure 1a,b). This allele was also found in another two compound heterozygous Caucasian GSD IIIA patients resident in the UK and the US, respectively (Table 2). All Faroese patients were homozygous for the same haplotype defined by five intragenic polymorphisms (Table 1), two of which (IVS 8-18 G A, IVS $23-$ $21 \mathrm{~T} \sim \mathrm{A})$ have not been previously reported. Results of haplotype analysis in the two compound heterozygotes who did not live in the Faroe Islands are compatible with the assumption that their c.1222T allele is also associated with the same polymorphisms (data not shown).

\section{Prevalence study}

The novel $A G L$ c.1222 C > T mutation was not detected in German newborns; in contrast, nine of 272 Faroese newborns were heterozygous (Table 2). These numbers predict a carrier frequency of $1: 30$ and a prevalence of one GSD IIIA case per 3600 individuals (95\% confidence interval 1:700-1:6400).

\section{Discussion}

In this study we report the molecular basis of GSD IIIA in patients from the Faroe Islands. Based upon haplotype analysis, we speculate that due to a founder effect in the small isolated population of the islands a regional cluster has

Table 1 Polymerase chain reaction primers used in this study for the formation of exon-specific amplimers and flanking intron regions of the $A G L$ gene. PCR products were subject to direct sequencing and/or to mutation/polymorphism detection by mutation- or polymorphism-specific restriction enzyme digestion. Ref., denotes the first report of a polymorphism. Sense (sn) and antisense (asn) primers were chosen from the published sequence with lowercase and uppercase letters representing intron and exon sequences, respectively. An underlined letter represents a mismatch. $T_{a}$, annealing temperature; RE, restriction enzyme. The haplotype associated with the c.1222T allele is highlighted in grey

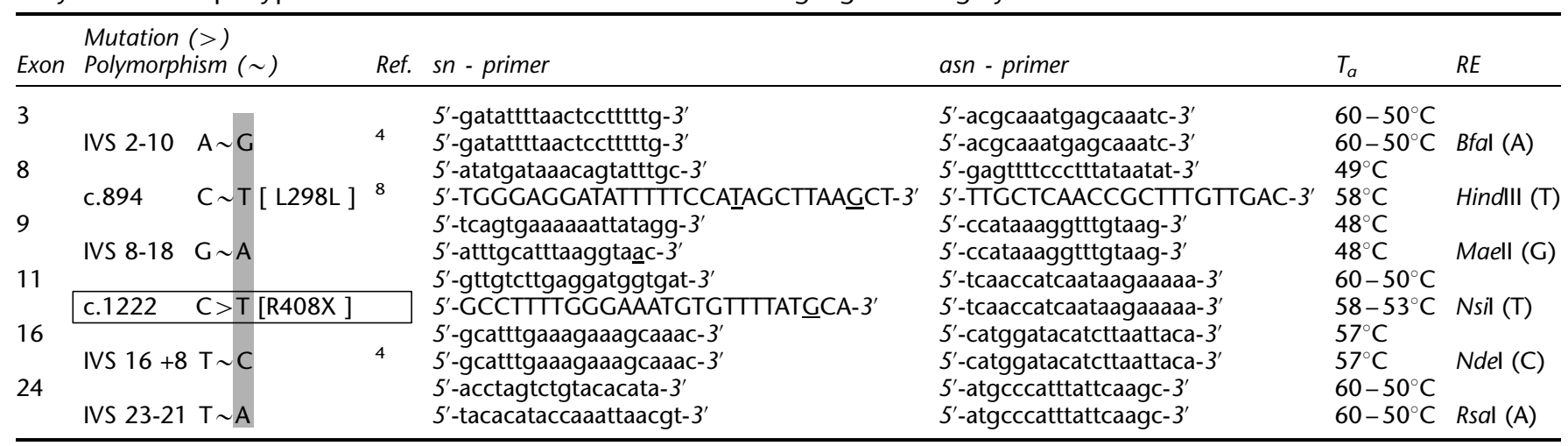


developed. The novel c.1222 $\mathrm{C}>\mathrm{T}$ mutation could be detected in six children investigated, and it is most likely also responsible for previously reported cases. ${ }^{3}$ It is interesting that also from the west coast of Norway where the Faroese population is supposed to have its origin, a cluster of GSD III

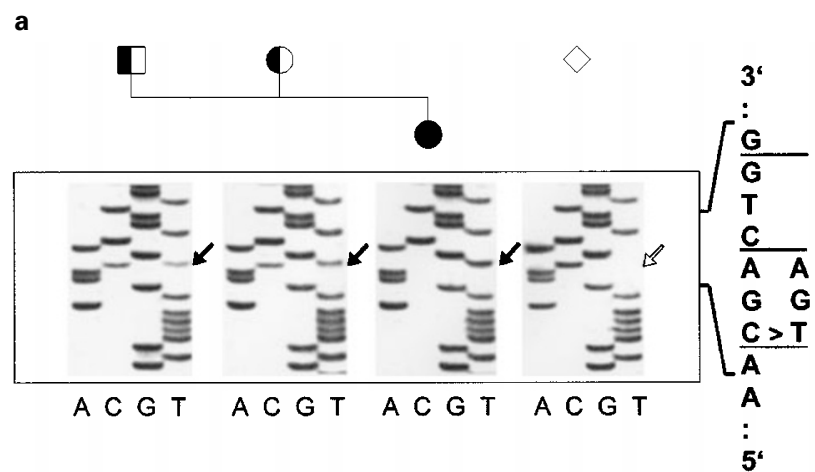

b
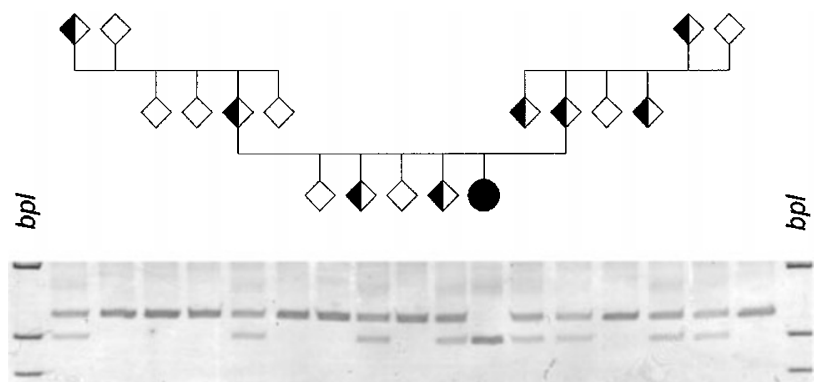

Figure 1 a Detection of the c.1222 C>T mutation (resulting in the replacement of a CGA codon (Arg) by a TGA codon (Stop)) by direct sequencing of a PCR product including exon 11 of the $A G L$ gene in the index family. $b$ Detection of the c.1222 $\mathrm{C}>\mathrm{T}$ mutation by restriction enzyme digest in the same family. Polyacrylamide gel electrophoresis of a mismatch PCR product treated with Nsil. bpl, base pair ladder. (Note that the genotype of individual members of the family cannot be deduced from this pedigree since the order of presentation within each generation was randomly chosen.)

Table 2 Frequency of the c.1222T allele of GSD IIIA among individuals from different neonatal screening programs and among unselected GSD IIIA cases of different origin. $\left({ }^{*} \chi^{2}=4.843 \quad(P=0.026)\right.$, †including cases from consanguineous families)

\begin{tabular}{|c|c|c|c|}
\hline & \multicolumn{2}{|c|}{ Neonatal screening } & $\begin{array}{l}\text { Unselected } \\
\text { GSD IIIA cases } \\
\text { Durham, NC }(\mathrm{n}=43) \\
\text { Kiel, Germany }(\mathrm{n}=7)\end{array}$ \\
\hline Positive & 0 * & 9* & 2 \\
\hline Negative & $396^{*}$ & $535^{*}$ & $98 \dagger$ \\
\hline Tested & 396 & 544 & $100 \dagger$ \\
\hline
\end{tabular}

cases has been reported $^{5}$ and it will be interesting to investigate the molecular basis of these cases.

There is evidence that the novel nonsense mutation in exon 11 is disease-causing; it results in truncation of the gene product which should affect both enzymatic activities of the AGL protein, oligo-1,4-1,4-glucantransferase and amylo-1,6glucosidase, due to the fact that the glycogen-binding site has been approximately located to a region encoded by exons 30 and $31 .^{6}$ Patients with less extensive carboxyterminal truncations have been shown to have characteristic clinical and biochemical features of GSD IIIA. ${ }^{1}$

The c.1222 C>T mutation is not confined to the Faroe Islands, and our results show that it plays a role, albeit minor, world-wide. The c.1222T allele was found in approximately $2 \%$ of alleles of unselected GSD III cases. However, most $A G L$ mutations are private and only four alleles (R864X, R1228X, c.3964 del T, and IVS32-12 A> G) have been found in more than $5 \%$ of unselected GSD III cases. ${ }^{1}$

We are currently aware of another eight cases of GSD IIIA in the Faroe Islands. This gives a total of 14 cases in a population of 45000 . From these figures, the observed prevalence of GSD IIIA in the Faroe Islands is $1: 3100$ which is within the same order of magnitude as 1:2000 (seven of 14000) reported previously. $^{3}$ These numbers are in agreement with the prevalence determined by measuring allele frequencies in neonates. This method rendered a prevalence of $1: 3600$ which is more than 30 times higher than the prevalence in an unselected population. ${ }^{7}$ It is the highest prevalence number for GSD IIIA world-wide, even when compared to another local cluster: for North African Jews in Israel a prevalence of $1: 5400$ for homozygosity for the c. $4455 \mathrm{del} \mathrm{T}$ allele has been reported. $^{2}$

The detection of the molecular defect has facilitated the diagnosis of GSD IIIA in the Faroe Islands. For example, in one of the patients reported in this study, diagnosis on peripheral leukocyte DNA and the introduction of appropriate treatment were possible in the neonatal period obviating the need for a liver biopsy. Knowledge of the basic defect will offer a better basis for genetic counselling of couples at risk and for first trimester prenatal diagnosis by direct genomic analysis. This may cause prevalence rates to decline in populations such as that of the cluster region in Israel (S. Moses, personal communication). So far, however, none of the Faroese families have requested prenatal diagnosis.

\section{Acknowledgments}

We wish to express our gratitude to the colleagues in the National Hospital Tórshavn, Faroe Islands, who cared for the patients, to I. Maire, Hôpital Debrousse, Lyon, for determination of glycogen content and amyloglucosidase activity in a liver sample, and to $A$. Kohlschütter, Hamburg, who provided Guthrie testcards from the Northern German screening program for inborn metabolic diseases. 


\section{References}

1 Shaiu WL, Kishnani PS, Shen J, Liu HM, Chen YT: Genotypephenotype correlation in two frequent mutations and mutation update in type III glycogen storage disease. Mol Genet Metab 2000; 69: 16-23.

2 Parvari R, Moses S, Shen J, Hershkovitz E, Lerner A, Chen YT: A single-base deletion in the 3 '-coding region of glycogendebranching enzyme is prevalent in glycogen storage disease type IIIA in a population of North African Jewish patients. Eur J Hum Genet 1997; 5: 266-270.

3 Cohn J, Wang P, Hauge M, Henningsen K, Jensen B, Svejgaard A: Amylo-1,6-glucosidase deficiency (glycogenosis type III) in the Faroe Islands. Hum Hered 1975; 25: $115-126$.

4 Okubo M, Horinishi A, Takeuchi M et al: Heterogeneous mutations in the glycogen-debranching enzyme gene are responsible for glycogen storage disease type IIIa in Japan. Hum Genet 2000; 106: $108-115$.
5 Moe PJ, Garatun-Tjeldstø E, Garatun-Tjeldstø O, Raae AF, Vogt $\mathrm{H}$ : Glycogen storage disease in Norway. Acta Paediatr Scand 1972; 61: 536-543.

6 Yang BZ, Ding JH, Enghild JJ, Bao Y, Chen YT: Molecular cloning and nucleotide sequence of cDNA encoding human muscle glycogen debranching enzyme. J Biol Chem 1992; 267: $9294-$ 9299.

7 Chen YT, Burchell A: Glycogen storage disease; in Scriver CR, Beaudet AL, Sly WS, Valle D (eds): The metabolic and molecular bases of inherited disease. McGraw-Hill, NewYork 1995, vol 1: pp 935-965.

8 Shen J, Liu HM, Bao Y, Chen YT: Polymorphic markers of the glycogen debranching enzyme gene allowing linkage analysis in families with glycogen storage type III. J Med Genet 1997; 34: $34-38$. 